\title{
Hepcidin Plays a Key Role in 6-OHDA Induced Iron Overload and Apoptotic Cell Death in a Cell Culture Model of Parkinson's Disease
}

\author{
Qi Xu, ${ }^{1,2}$ Anumantha G. Kanthasamy, ${ }^{3}$ Huajun Jin, ${ }^{3}$ and Manju B. Reddy ${ }^{1}$ \\ ${ }^{1}$ Department of Food Science and Human Nutrition, Iowa State University, Ames, IA 50011, USA \\ ${ }^{2}$ School of Public Health, Shanghai University of Traditional Chinese Medicine, Shanghai 201203, China \\ ${ }^{3}$ Department of Biomedical Sciences, Iowa State University, Ames, IA 50011, USA
}

Correspondence should be addressed to Manju B. Reddy; mbreddy@iastate.edu

Received 10 February 2016; Accepted 5 May 2016

Academic Editor: Ivan Bodis-Wollner

Copyright (C) 2016 Qi Xu et al. This is an open access article distributed under the Creative Commons Attribution License, which permits unrestricted use, distribution, and reproduction in any medium, provided the original work is properly cited.

Background. Elevated brain iron levels have been implicated in the pathogenesis of Parkinson's disease (PD). However, the precise mechanism underlying abnormal iron accumulation in PD is not clear. Hepcidin, a hormone primarily produced by hepatocytes, acts as a key regulator in both systemic and cellular iron homeostasis. Objective. We investigated the role of hepcidin in 6hydroxydopamine (6-OHDA) induced apoptosis in a cell culture model of PD. Methods. We downregulated hepcidin using siRNA interference in N27 dopaminergic neuronal cells and made a comparison with control siRNA transfected cells to investigate the role of hepcidin in 6-OHDA induced neurodegeneration. Results. Hepcidin knockdown $(32.3 \%, P<0.0001)$ upregulated ferroportin 1 expression and significantly $(P<0.05)$ decreased intracellular iron by $25 \%$. Hepcidin knockdown also reduced 6-OHDA induced caspase- 3 activity by $42 \%(P<0.05)$ and DNA fragmentation by $29 \%(P=0.086)$ and increased cell viability by $22 \%(P<0.05)$. In addition, hepcidin knockdown significantly attenuated 6-OHDA induced protein carbonyls by 52\% $(P<0.05)$ and intracellular iron by $28 \%(P<0.01)$, indicating the role of hepcidin in oxidative stress. Conclusions. Our results demonstrate that hepcidin knockdown protected N27 cells from 6-OHDA induced apoptosis and that hepcidin plays a major role in reducing cellular iron burden and oxidative damage by possibly regulating cellular iron export mediated by ferroportin 1 .

\section{Introduction}

Parkinson's disease (PD) is an incurable neurodegenerative disease that affects more than $1 \%$ of people over 65 years old and approximately $4 \%$ of the population aged over 80 years [1]. The prevalence is expected to rise sharply within the next two decades because of progressive aging population [2]. Parkinson's disease is characterized by the progressive loss of dopaminergic neurons in the substantia nigra (SN), degeneration of projecting nerve fibers in the striatum, and accumulation of intracytoplasmic inclusions, known as Lewy bodies [3]. Although the etiology of PD is not clear, both genetic and environmental risk factors including exposure to metals and pesticides are considered to be involved in PD [4].

Iron, the most abundant trace metal in the brain, is thought to play an important role in the pathogenesis of
PD. Studies have demonstrated the association between iron dysregulation and PD. Increased levels of iron deposits in the SN are observed in postmortem studies [5], as well as in 6-hydroxydopamine (6-OHDA) [6] and 1-methyl-4phenyl-1,2,3,6-tetrahydropyridine (MPTP) [7] induced PD animal models. The imaging studies of living PD patients also confirmed the presence of accumulation of iron in the SN and linked the extent of iron deposits to the severity of disease [8]. Although iron is important in various physiological functions, such as DNA synthesis, mitochondrial respiration, and oxygen transport [9], free iron is potentially toxic as it is involved in the generation of hydroxyl radicals, which can react with lipid, protein, and DNA, leading to subsequent neuronal damage and death. Moreover, free iron in dopaminergic neurons can accelerate toxic alpha-synuclein fibril formation, leading to neuronal dysfunction [10]. 
Because of the potential toxicity of iron, iron homeostasis is tightly regulated by a complex system that coordinates iron uptake, release, storage, and utilization. For example, iron is delivered to tissues by circulating transferrin, and excess iron in the cell is stored in the cytosolic ferritin [11]. Hepcidin is a small peptide that is mainly secreted by hepatocytes in response to inflammation, iron overload, and oxidative stress $[12,13]$. It controls systemic iron levels by regulating iron absorption from the intestine, the release of iron from degraded hemoglobin from macrophages, and stored iron from hepatocyte [14]. Hepcidin is also considered as a master regulator in the management of cellular iron homeostasis by binding to iron exporter protein ferroportin 1 (Fpn1) in cell membranes and causing its subsequent internalization and lysosomal degradation [3]. Although hepcidin is predominantly expressed in the liver, recent research demonstrates that hepcidin is also widely distributed in the central nervous system. One study showed that hepcidin mRNA level is increased with aging in mouse brain, particularly in the cerebral cortex, hippocampus, and striatum, which leads to decreased level of Fpn1 and the associated iron accumulation in aging brain [15]. Another study showed that peripheral iron overload induces hepcidin and decreased level of Fpn1 in the SN of rats, suggesting the critical role that hepcidin plays in brain iron disturbance [16]. The objective of this study was to determine the role of hepcidin in 6-OHDA induced cell death by knocking down hepcidin expression in N27 dopaminergic cell model of PD.

\section{Materials and Methods}

2.1. Chemicals. The immortalized rat mesencephalic dopaminergic neuronal cell line (1RB3AN27, generally referred to as N27) was a kind gift from Dr. Kedar N. Prasad, University of Colorado Health Sciences Center (Denver, CO). RPMI1640 medium, fetal bovine serum, L-glutamine, penicillin, and streptomycin were obtained from Invitrogen (Carlsbad, CA). Calcein-AM, ascorbic acid, mouse $\beta$-actin antibody, 6 OHDA, ferrous sulfate, and ascorbic acid were purchased from Sigma-Aldrich (St. Louis, MO). The Amaxa Nucleofector kit was purchased from Lonza (Allendale, NJ). The Absolutely RNA Miniprep kit and High Capacity cDNA Archive kit were purchased from Stratagene (La Jolla, CA) and Life Technologies (Grand Island, NY), respectively. The hepcidin specific siRNA and scrambled siRNA were purchased from Integrated DNA Technologies (Coralville, IA). Substrate for caspase-3, acetyl-Asp-Glu-Val-Asp-AFC, was obtained from MP Biomedicals (Solon, OH). The Cell Death Detection ELISA Plus kit was purchased from Roche Diagnostics (Indianapolis, IN). Protein Carbonyls Colorimetric Assay kit was purchased from Cayman Chemical (Ann Arbor, MI). The rabbit polyclonal antibody for Fpn1 was purchased from Alpha Diagnostic (San Antonio, TX). Alexa Fluor 680 conjugated anti-mouse IgG and IR-dye 800 conjugated anti-rabbit IgG were purchased from Invitrogen (Carlsbad, CA) and Rockland Inc. (Gilbertsville, PA), respectively. All solutions were prepared fresh prior to each assay.
2.2. Cell Culture. N27 cells were grown in RPMI-1640 medium containing $10 \%$ fetal bovine serum, $2 \mathrm{mmol} / \mathrm{L} \mathrm{L}$ glutamine, 50 units of penicillin, and $50 \mu \mathrm{g} / \mathrm{mL}$ streptomycin and maintained at $37^{\circ} \mathrm{C}$ in a humidified atmosphere containing $5 \% \mathrm{CO}_{2}$, as described in our previous publication [17].

2.3. Transient Transfections and Treatment Paradigm. N27 cells were transfected with hepcidin specific siRNA (hepcidin siRNA) or scrambled siRNA (control siRNA) using the Amaxa Nucleofector kit, following the manufacturer's instructions. Briefly, $3 \times 10^{6}$ cells were resuspended in $100 \mu \mathrm{L}$ of the Nucleofector solution, along with $1.5 \mu \mathrm{g}$ of hepcidin siRNA or control siRNA, and subsequently subjected to electroporation using the Nucleofector program number A23. After 72 hours of initial transfection, cells were harvested and hepcidin mRNA was analyzed using quantitative real-time RT-PCR to confirm the knockdown efficiency. To evaluate the effect of hepcidin knockdown on 6-OHDA induced neurotoxicity, both control siRNA and hepcidin siRNA transfected cells were plated for 48 hours and treated with or without $100 \mu \mathrm{M} 6$-OHDA for 6 hours. Cells were collected at the end of each treatment for the following experiments.

2.4. Quantitative Real-Time RT-PCR. Total RNA was isolated and converted to cDNA using the Absolutely RNA Miniprep kit and High Capacity cDNA Archive kit, respectively. Realtime PCR was performed using a Brilliant SYBR Green QPCR Master Mix kit and the Mx3000P QPCR system, as described in our previous publication [18]. The 18s rRNA was used as an internal control for quantifying RNA with the primer set purchased from SABiosciences (Valencia, CA). The reaction mixture included $2 \mu \mathrm{L}$ of cDNA, $12.5 \mu \mathrm{L}$ of $2 x$ master mix, and $0.2 \mu \mathrm{mol} / \mathrm{L}$ of each primer. Cycling conditions contained initial denaturation at $95^{\circ} \mathrm{C}$ for $10 \mathrm{~min}$, followed by 40 cycles of denaturation at $95^{\circ} \mathrm{C}$ for $15 \mathrm{~s}$ and annealing at $60^{\circ} \mathrm{C}$ for $10 \mathrm{~min}$. Fluorescence was detected during the annealing/extension step of each cycle. Dissociation curves were run to verify the singularity of the PCR products. The data were analyzed using the comparative threshold cycle method as described in our previous publication [18].

2.5. Western Blot Assay for Fpn1. Cell lysates were prepared using a modified radio immunoprecipitation assay (RIPA) buffer as described previously [19]. Equal amounts of protein were loaded for each sample and separated on 12\% SDSPAGE gels. After separation, the proteins were transferred onto a nitrocellulose membrane and were incubated with the rabbit polyclonal antibody directed against Fpn1 $(1: 1000)$ and developed with IR-dye 800 anti-rabbit secondary antibody (1:5000). $\beta$-actin was used as the loading control. Membranes were visualized on an Odyssey Infrared Imaging system (LICOR, Lincoln, NE).

2.6. Calcein Quenching to Measure Intracellular Iron Levels. The intracellular iron levels were determined by a calcein fluorescence quenching method modified from a previous study [20]. Calcein-AM is a membrane permeable, nonfluorescent molecule that becomes fluorescent by intracellular 
esterases. It is quenched rapidly by $\mathrm{Fe}^{2+}$ or $\mathrm{Fe}^{3+}$ and is a good indicator of the labile iron pool [20], which is cellular free iron or cheatable iron. The degree of quenching gives an estimate of the amounts of cellular cheatable iron. After the treatment, cells were incubated with calcein-AM in HEPESbuffered saline (HBS) for $30 \mathrm{~min}$ at $37^{\circ} \mathrm{C}$. The excess calcein on the cell surface was washed off three times with HBS, and fluorescence was recorded using a Synergy II microplate reader (BioTek Instruments, Winooski, VT) at $485 \mathrm{~nm}$ excitation and $530 \mathrm{~nm}$ emission. Change in fluorescence intensity (with and without treatment after normalizing to protein concentrations) reflected the intracellular iron levels. Calcein fluorescence pictures were obtained with FLoid ${ }^{\circledR}$ Cell Imaging Station (Life Technologies).

2.7. Cell Viability Assays. Cell viability was measured using MTT assay as described earlier [17]. After each treatment, cells were incubated with serum-free RPMI medium containing $0.25 \mathrm{mg} / \mathrm{mL}$ MTT solution for $3 \mathrm{~h}$ at $37^{\circ} \mathrm{C}$, followed by adding isopropanol- $\mathrm{HCl}(200 \mu \mathrm{L})$ solution to dissolve intracellular purple formazan. The absorbance was read at $570 \mathrm{~nm}$ with a reference wavelength of $630 \mathrm{~nm}$ using a microplate reader (Molecular Devices, Sunnyvale, CA).

2.8. Caspase-3 Activity Assays. Caspase-3 activity was measured as described previously [21]. After treatment, the cell pellet after centrifugation was lysed with Tris buffer $(50 \mathrm{~mol} / \mathrm{L}$ Tris- $\mathrm{HCl}, 1 \mathrm{mmol} / \mathrm{L}$ EDTA, and $10 \mathrm{mmol} / \mathrm{L}$ EGTA at $\mathrm{pH}=7.4$ ) containing $10 \mu \mathrm{mol} / \mathrm{L}$ digitonin for $20 \mathrm{~min}$ at $37^{\circ} \mathrm{C}$. Lysates were subjected to quick centrifugation at $14,000 \times \mathrm{g}$ and then incubated with a specific fluorescent substrate (Ac-DEVDAFC, $50 \mu \mathrm{mol} / \mathrm{L}$ ) for $1 \mathrm{~h}$ at $37^{\circ} \mathrm{C}$. The caspase- 3 activity was measured with excitation at $400 \mathrm{~nm}$ and emission at $505 \mathrm{~nm}$ using a fluorescence microplate reader. The caspase-3 activity was expressed as fluorescent units/mg protein.

2.9. DNA Fragmentation Assays. DNA fragmentation assays were performed using the Cell Death Detection ELISA Plus kit as described previously [19]. After treatment, cell pellet was incubated with lysis buffer provided in the kit. The lysates were then centrifuged and the supernatant was incubated for $2 \mathrm{~h}$ with the mixture of HRP-conjugated antibody cocktail that recognizes histones and single- and double-stranded DNA. After washing away the unbound components, measurements were made at $490 \mathrm{~nm}$ and $405 \mathrm{~nm}$ using a fluorescence microplate reader. DNA fragmentation was expressed as absorbance units/mg protein.

2.10. Protein Carbonyl Assays. The oxidative damage to proteins was determined by measuring the protein carbonyl residues using DNPH (2,4-dinitrophenylhydrazine) according to the manufacturer's protocol. DNPH reacts with protein carbonyls to produce the corresponding hydrazones, which was measured spectrophotometrically at the wavelength of $360 \mathrm{~nm}$. The carbonyl content was determined from the differences in absorbance between DNPH-reacted samples and nonreacted $\mathrm{HCl}$ samples and then standardized against the protein concentrations in the samples.
2.11. Statistical Analysis. Data were analyzed using the GraphPad Prism 5.0 (GraphPad Software, Inc., La Jolla, CA). All values were expressed as mean \pm SEM. Student's $t$-test was used to compare the differences between groups. The values for two (control and hepcidin) siRNA transfected cells with 6-OHDA treatments were normalized to their respective controls (without 6-OHDA treatment) before statistical analysis. All the mean differences were considered significant at $P \leq$ 0.05 .

\section{Results}

3.1. Downregulation of Hepcidin. To address the role of hepcidin in regulation of 6-OHDA induced neurotoxicity, we first utilized RNA interference (RNAi) technique to downregulate hepcidin levels in N27 dopaminergic cells. As shown in Figure 1(a), hepcidin mRNA levels were significantly downregulated $(32.3 \%, P<0.0001)$ in hepcidin siRNA transfected cells compared with control siRNA transfected cells. Since hepcidin regulates Fpn1 by triggering its degradation, we further determined whether downregulation of hepcidin leads to increased Fpnl protein expression (Figure 1(b)). Compared to the control siRNA transfected cells, the Fpnl protein levels were elevated in hepcidin siRNA transfected cells, which confirmed a negative relationship between hepcidin and Fpnl expression in dopaminergic cells. We also measured intracellular iron, which was indirectly measured by calcein fluorescence quenching, to ascertain whether decreased expression of hepcidin and increased expression of Fpn1 reduced intracellular iron levels. Calcein is the most widely adopted iron fluorescent probe that has been used to monitor changes of iron levels in a range of different cell types such as hepatocytes and colon cells [22]. As shown in Figure 1(c), hepcidin knockdown significantly decreased intracellular iron by $25 \%(P<0.05)$. To confirm calcein fluorescence quenching by intracellular iron, we incubated the cells with or without $1 \mathrm{mM}$ exogenous iron (ferrous sulfate in ascorbic acid solution, 1:44 molar ratio, $\mathrm{pH}$ 6.0) for $30 \mathrm{~min}$ and then examined the calcein quenching by fluorescence microscopy. As shown in Figure 1(d), supplementation of $1 \mathrm{mM}$ ferrous sulfate effectively decreased calcein fluorescence.

3.2. Hepcidin Knockdown Protects N27 Cells from 6-OHDA Induced Cytotoxicity. All the values in Figure 2 were presented as percentage of respective controls without 6-OHDA treatment. We evaluated the effect of hepcidin knockdown on 6-OHDA mediated cell death using MTT assay (Figure 2(a)). Hepcidin knockdown moderately but significantly lessened the toxic effect of 6-OHDA by increasing cell viability by $22 \%(P<0.05)$. When apoptosis was measured, hepcidin knockdown reduced 6-OHDA induced caspase-3 activity significantly (Figure 2(b), 42\%, $P<0.05$ ). DNA fragmentation was also reduced, but it was only marginally significant (Figure 2(c), $P=0.086)$. Together, these results demonstrate that hepcidin knockdown protects against 6-OHDA induced cell apoptosis.

3.3. Hepcidin Downregulation Reduces 6-OHDA Induced Protein Oxidative Damage and Intracellular Iron. As shown in 


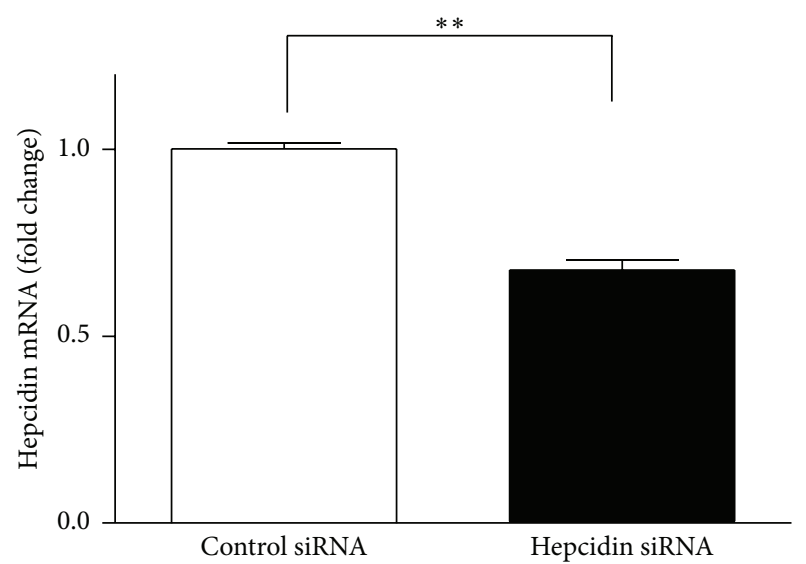

(a)

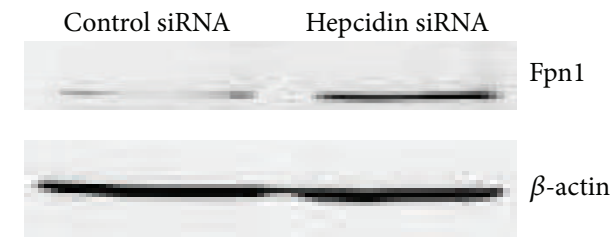

(b)

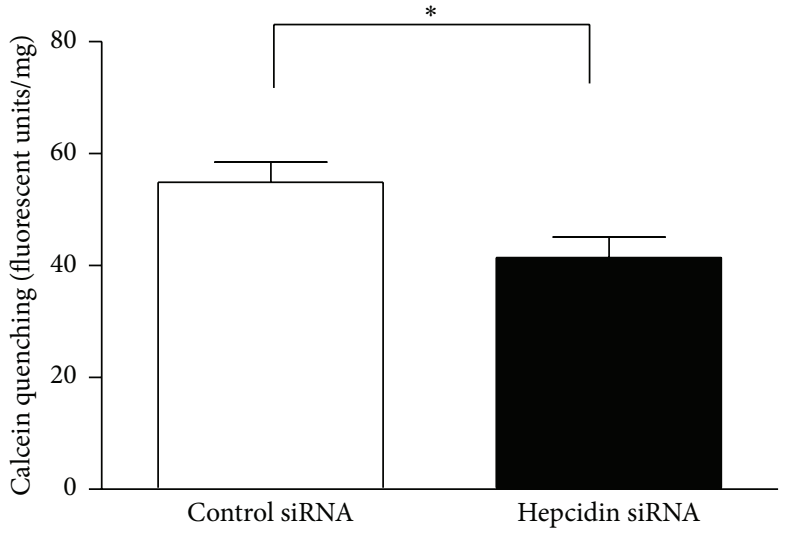

(c)

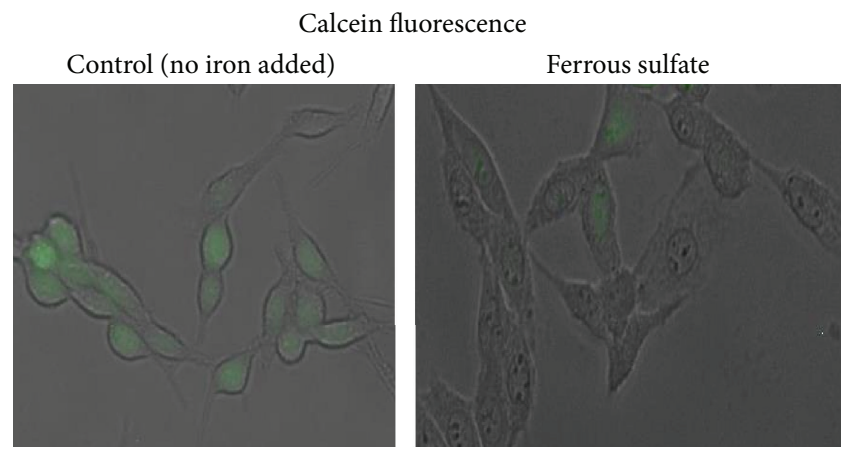

(d)

FIGURE 1: Effect of hepcidin knockdown on hepcidin mRNA levels measured by quantitative real-time RT-PCR ((a), $n=7-8)$, ferroportin 1 protein levels (normalized to $\beta$-actin) measured by Western blot (b), and intracellular iron measured by a calcein quenching method ((c), $n=6)$ in N27 cells. Representative calcein fluorescence images with and without incubation of $1 \mathrm{mM}$ ferrous sulfate for $30 \mathrm{~min}$ are shown (d). Values are mean \pm SEM. Differences between two groups were based on Student's $t$-test; ${ }^{*} P<0.05 ;{ }^{* *} P<0.0001$. Control siRNA: scrambled small interfering RNA; hepcidin siRNA: hepcidin small interfering RNA; Fpnl: ferroportin 1.

TABLE 1: The role of hepcidin knockdown in 6-OHDA induced oxidative damage measured by protein carbonyls $(n=4)$ and intracellular iron measured by calcein quenching method $(n=6)$.

\begin{tabular}{lcc}
\hline & Protein carbonyls (nmol/mg protein) & Calcein quenching (fluorescent units/mg protein) \\
\hline Control siRNA & $18.5 \pm 2.9$ & $112.4 \pm 4.8$ \\
Hepcidin siRNA & $8.9 \pm 1.1^{*}$ & $80.8 \pm 6.2^{* *}$ \\
\hline
\end{tabular}

Values are mean $\pm \mathrm{SEM} ;{ }^{*} P<0.05 ;{ }^{* *} P<0.01$. Differences between two groups were based on Student's $t$-test; control siRNA: scrambled small interfering RNA; hepcidin siRNA: hepcidin small interfering RNA.

Table 1, hepcidin knockdown decreased 6-OHDA induced protein carbonyls by 52\% $(P<0.05)$ and intracellular iron by $28 \%(P<0.01)$. These results show that hepcidin knockdown might protect against 6-OHDA induced neurotoxicity through attenuating oxidative stress by mediating intracellular free iron.

\section{Discussion}

Iron is an essential nutrient and is involved in many functions, such as acting as a cofactor for key enzymes involved in neurotransmitter biosynthesis [23]. On the other hand, excess free iron can cause significant oxidative stress by involvement in the production of hydroxyl radical formation, glutathione consumption, protein aggregation, lipid peroxidation, and nucleic acid modification $[24,25]$. The body iron homeostasis is regulated by iron regulatory proteins to minimize the amount of free iron available to participate in free radical formation. Among those proteins, hepcidin is considered as a principal regulator because of its function to inhibit cellular efflux of iron by binding to Fpnl at the cell surface and inducing its subsequent degradation [26].

Recent studies have suggested a critical role for hepcidin in a variety of disorders, including anemia of inflammation, 


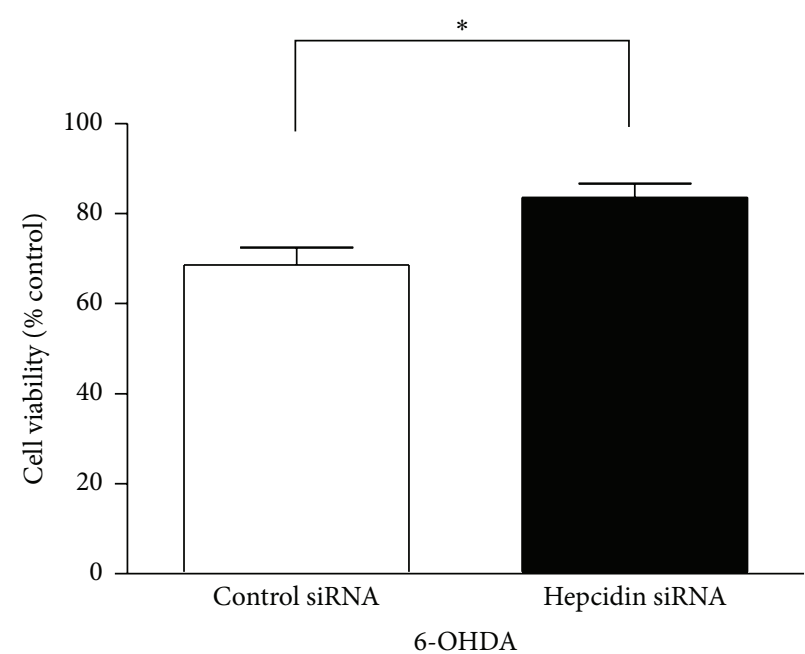

(a)

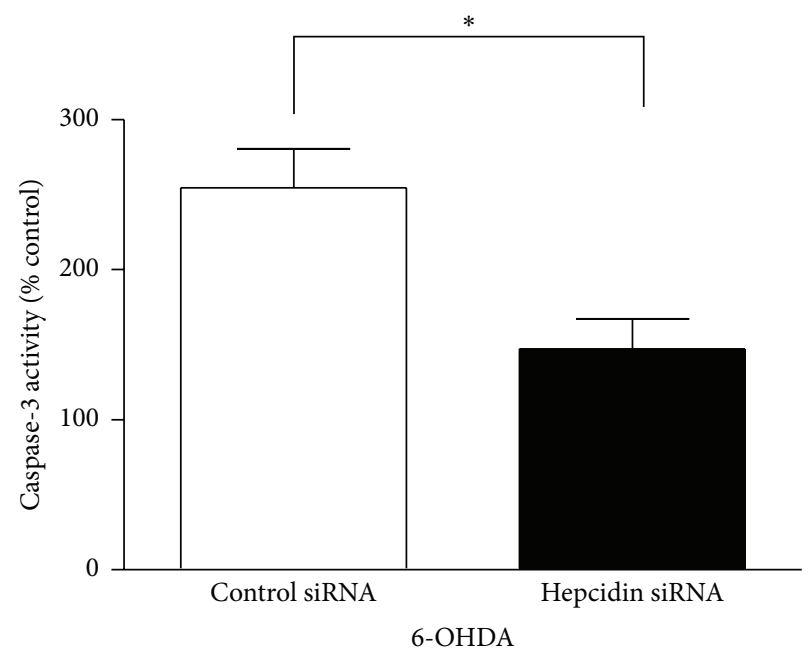

(b)

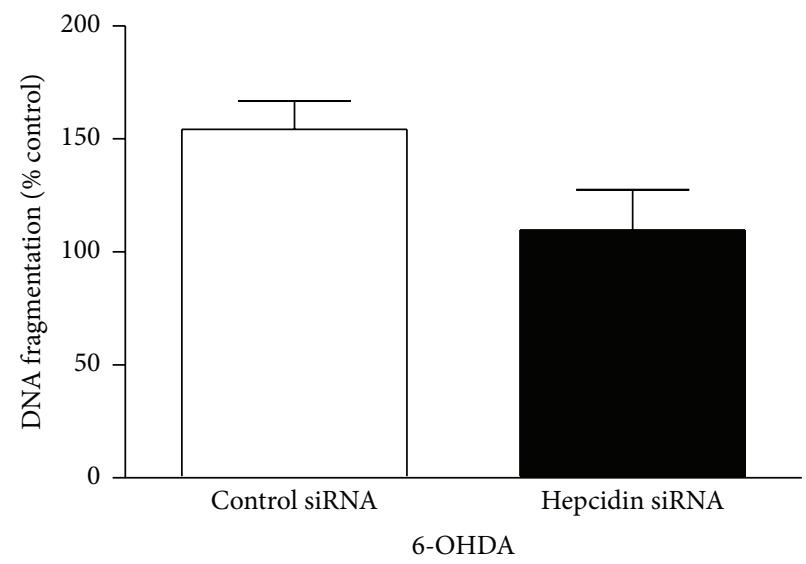

(c)

FIGURE 2: The role of hepcidin knockdown in 6-OHDA induced cytotoxicity measured by MTT ((a), $n=6)$, caspase-3 activity ((b), $n=4-5)$, and DNA fragmentation $((\mathrm{c}), n=4)$ in N27 cells. Cells were treated with $100 \mu \mathrm{M}$ 6-OHDA for 6 hours and the values (mean \pm SEM) are normalized to their respective controls without 6-OHDA treatment; ${ }^{*} P<0.05$; difference between two groups was based on Student's $t$-test; control siRNA: scrambled small interfering RNA; hepcidin siRNA: hepcidin small interfering RNA.

chronic kidney disease, and familial hemochromatosis [2628]. However, the participation of hepcidin in neurodegenerative disorders is very limited. In our earlier study on cell culture [29], 6-OHDA increased the expression of hepcidin and decreased the expression of Fpnl, which made us design this current study to investigate the effect of hepcidin with knockdown experiments. We used N27 dopaminergic neuronal cell model to detect hepcidin and Fpn1 expression, since N27 cell line possesses all physiological and biochemical properties of dopaminergic neurons [30]. Our results show that both hepcidin and Fpnl are expressed in N27 cells and that knockdown of hepcidin remarkably increased Fpnl expression and reduced intracellular iron levels as measured by calcein quenching. These results are consistent with previous studies, which demonstrate that hepcidin is widely expressed in murine brain and might play a key role in regulating iron levels in the brain by downregulating Fpnl expression [15, 31, 32].
Our study also shows that regulation of brain iron efflux by hepcidin may play a protective role in 6-OHDA induced neurotoxicity. Hepcidin knockdown and subsequent upregulation of Fpnl protein significantly attenuated the protein oxidative damage induced by 6-OHDA, ultimately leading to a reduction in cell apoptosis, as evidenced by decreased caspase- 3 activation and marginally decreasing DNA fragmentation. Increasing caspase-3 activity and DNA fragmentation, respectively, more than 2 - and 1.5 -fold in the control siRNA cells but only showing a small increase in hepcidin siRNA cells with 6-OHDA treatment clearly shows the protection with hepcidin downregulation, even with low knockdown efficiency. 6-OHDA is a hydroxylated analogue of the neurotransmitter dopamine and represents a classic neurotoxin used for the initiation of the PD neurodegeneration both in vitro and in vivo [33]. Studies have shown abnormal iron accumulation in 6-OHDA induced PD models, and 6-OHDA induced neurotoxicity may result 
from free iron and the ensuing production of free radical species [34]. However, the precise mechanism underlying abnormal iron accumulation in 6-OHDA induced neurotoxicity is not very clear. Song et al. [35] demonstrated that upregulation of iron regulatory protein 1 (IRP1) might be responsible for decreased expression of Fpnl and increased cellular iron accumulation. Another study reported that divalent metal transporter 1 (DMT1) + IRE upregulation is involved in 6-OHDA induced iron accumulation and aggravated oxidative injury [36]. Our study provides direct evidence for the first time that hepcidin-ferroportin axis at least partially accounts for iron accumulation in 6-OHDA induced neurodegeneration. Hepcidin knockdown resulted in upregulation of Fpnl, which may enhance iron release and alleviate iron accumulation in dopaminergic neurons, and eventually protected neurons from 6-OHDA induced apoptosis. Our data with calcein quenching support this relationship. In addition to its role in iron homeostasis, hepcidin is also recognized as a principal mediator in inflammation $[37,38]$, which is also directly linked to the pathogenesis of PD $[39,40]$. Thus, further study is needed to study the linkage between hepcidin expression, iron status, and neuroinflammation in PD. In addition, research has shown that 6-OHDA can induce oxidative damage and neurotoxicity of both the peripheral and the central nervous system [41], and hepcidin is strongly expressed in other brain regions such as the olfactory bulb [42]. Future research is needed to investigate whether hepcidin has a role in protecting from 6-OHDA induced neurotoxicity in other brain regions.

\section{Conclusions}

In conclusion, our study demonstrates that hepcidin plays an important role in iron accumulation, thus causing oxidative stress and associated neurotoxicity. Hence, the approaches that can reduce hepcidin and increase Fpnl expression might be effective strategies in preventing the progression of PD.

\section{Abbreviations}

PD: $\quad$ Parkinson's disease

6-OHDA: 6-Hydroxydopamine

SN: $\quad$ Substantia nigra

MPTP: 1-Methyl-4-phenyl-1,2,3,6-tetrahydropyridine

Fpnl: $\quad$ Ferroportin 1

RIPA: Radio immunoprecipitation assay

HBS: $\quad$ HEPES-buffered saline

DNPH: 2,4-Dinitrophenylhydrazine

RNAi: RNA interference

IRP1: Iron regulatory protein 1

DMT1: Divalent metal transporter 1

IRE: Iron responsive element.

\section{Competing Interests}

The authors have no competing interests to declare.

\section{Acknowledgments}

This study is supported by the College of Human Sciences, Iowa State University.

\section{References}

[1] H. Diao, X. Li, S. Hu, and Y. Liu, "Gene expression profiling combined with bioinformatics analysis identify biomarkers for parkinson disease," PLoS ONE, vol. 7, no. 12, Article ID e52319, 2012.

[2] S. Sharma, C. S. Moon, A. Khogali et al., "Biomarkers in Parkinson's disease (recent update)," Neurochemistry International, vol. 63, no. 3, pp. 201-229, 2013.

[3] O. Myhre, H. Utkilen, N. Duale, G. Brunborg, and T. Hofer, "Metal dyshomeostasis and inflammation in Alzheimer's and Parkinson's diseases: possible impact of environmental exposures," Oxidative Medicine and Cellular Longevity, vol. 2013, Article ID 726954, 19 pages, 2013.

[4] K. Wirdefeldt, H.-O. Adami, P. Cole, D. Trichopoulos, and J. Mandel, “Epidemiology and etiology of Parkinson's disease: a review of the evidence," European Journal of Epidemiology, vol. 26, supplement 1, pp. S1-S58, 2011.

[5] E. Sofic, P. Riederer, H. Heinsen et al., "Increased iron (III) and total iron content in post mortem substantia nigra of parkinsonian brain," Journal of Neural Transmission, vol. 74, no. 3, pp. 199-205, 1988.

[6] H. Jiang, N. Song, H. Xu, S. Zhang, J. Wang, and J. Xie, "Up-regulation of divalent metal transporter 1 in 6-hydroxydopamine intoxication is IRE/IRP dependent," Cell Research, vol. 20, no. 3, pp. 345-356, 2010.

[7] Z. Lv, H. Jiang, H. Xu, N. Song, and J. Xie, "Increased iron levels correlate with the selective nigral dopaminergic neuron degeneration in Parkinson's disease," Journal of Neural Transmission, vol. 118, no. 3, pp. 361-369, 2011.

[8] S. L. Rhodes and B. Ritz, "Genetics of iron regulation and the possible role of iron in Parkinson's disease," Neurobiology of Disease, vol. 32, no. 2, pp. 183-195, 2008.

[9] R. B. Mounsey and P. Teismann, "Chelators in the treatment of iron accumulation in parkinson's disease," International Journal of Cell Biology, vol. 2012, Article ID 983245, 12 pages, 2012.

[10] S. Oshiro, M. S. Morioka, and M. Kikuchi, "Dysregulation of iron metabolism in Alzheimer's disease, Parkinson's disease, and amyotrophic lateral sclerosis," Advances in Pharmacological Sciences, vol. 2011, Article ID 378278, 8 pages, 2011.

[11] J. Wang and K. Pantopoulos, "Regulation of cellular iron metabolism," Biochemical Journal, vol. 434, no. 3, pp. 365-381, 2011.

[12] T. Ganz, "Hepcidin-a regular of intestinal iron absorption and iron recycling by macrophages," Best Practice and Research: Clinical Haematology, vol. 18, no. 2, pp. 171-182, 2005.

[13] G. Millonig, I. Ganzleben, T. Peccerella et al., "Sustained submicromolar $\mathrm{H}_{2} \mathrm{O}_{2}$ levels induce hepcidin via signal transducer and activator of transcription 3 (STAT3)," The Journal of Biological Chemistry, vol. 287, no. 44, pp. 37472-37482, 2012.

[14] E. Nemeth, M. S. Tuttle, J. Powelson et al., "Hepcidin regulates cellular iron efflux by binding to ferroportin and inducing its internalization," Science, vol. 306, no. 5704, pp. 2090-2093, 2004.

[15] S.-M. Wang, L.-J. Fu, X.-L. Duan et al., "Role of hepcidin in murine brain iron metabolism," Cellular and Molecular Life Sciences, vol. 67, no. 1, pp. 123-133, 2010. 
[16] C. Sun, N. Song, A. Xie, J. Xie, and H. Jiang, "High hepcidin level accounts for the nigral iron accumulation in acute peripheral iron intoxication rats," Toxicology Letters, vol. 212, no. 3, pp. 276281, 2012.

[17] Q. Xu, A. G. Kanthasamy, and M. B. Reddy, "Neuroprotective effect of the natural iron chelator, phytic acid in a cell culture model of Parkinson's disease," Toxicology, vol. 245, no. 1-2, pp. 101-108, 2008.

[18] H. Jin, A. Kanthasamy, A. Ghosh, Y. Yang, V. Anantharam, and A. G. Kanthasamy, " $\alpha$-synuclein negatively regulates protein kinase $C \delta$ expression to suppress apoptosis in dopaminergic neurons by reducing p300 histone acetyltransferase activity," The Journal of Neuroscience, vol. 31, no. 6, pp. 2035-2051, 2011.

[19] R. Gordon, V. Anantharam, A. G. Kanthasamy, and A. Kanthasamy, "Proteolytic activation of proapoptotic kinase protein kinase $\mathrm{C} \delta$ by tumor necrosis factor $\alpha$ death receptor signaling in dopaminergic neurons during neuroinflammation," Journal of Neuroinflammation, vol. 9, article 82, 2012.

[20] W. Wang, N. Song, H. Zhang, J. Xie, and J. Wang, "6Hydroxydopamine upregulates iron regulatory protein 1 by activating certain protein kinase $\mathrm{C}$ isoforms in the dopaminergic MES23.5 cell line," International Journal of Biochemistry and Cell Biology, vol. 44, no. 11, pp. 1987-1992, 2012.

[21] F. Sun, A. Kanthasamy, C. Song, Y. Yang, V. Anantharam, and A. G. Kanthasamy, "Proteasome inhibitor-induced apoptosis is mediated by positive feedback amplification of $\mathrm{PKC} \delta$ proteolytic activation and mitochondrial translocation," Journal of Cellular and Molecular Medicine, vol. 12, no. 6, pp. 2467-2481, 2008.

[22] Y. Ma, V. Abbate, and R. C. Hider, "Iron-sensitive fluorescent probes: monitoring intracellular iron pools," Metallomics, vol. 7, no. 2, pp. 212-222, 2015.

[23] R. R. Crichton, D. T. Dexter, and R. J. Ward, "Brain iron metabolism and its perturbation in neurological diseases," Journal of Neural Transmission, vol. 118, no. 3, pp. 301-314, 2011.

[24] P. Urrutia, P. Aguirre, A. Esparza et al., "Inflammation alters the expression of DMT1, FPN1 and hepcidin, and it causes iron accumulation in central nervous system cells," Journal of Neurochemistry, vol. 126, no. 4, pp. 541-549, 2013.

[25] H. Yang, M. Yang, H. Guan et al., "Mitochondrial ferritin in neurodegenerative diseases," Neuroscience Research, vol. 77, no. 1-2, pp. 1-7, 2013.

[26] M. Franchini, M. Montagnana, and G. Lippi, "Hepcidin and iron metabolism: from laboratory to clinical implications," Clinica Chimica Acta, vol. 411, no. 21-22, pp. 1565-1569, 2010.

[27] E. Beutler, "Hemochromatosis: genetics and pathophysiology," Annual Review of Medicine, vol. 57, pp. 331-347, 2006.

[28] T. Ganz and E. Nemeth, "The hepcidin-ferroportin system as a therapeutic target in anemias and iron overload disorders," Hematology/The Education Program of the American Society of Hematology, vol. 2011, no. 1, pp. 538-542, 2011.

[29] D. Chen, A. G. Kanthasamy, and M. B. Reddy, "EGCG protects against 6-OHDA-induced neurotoxicity in a cell culture model," Parkinson's Disease, vol. 2015, Article ID 843906, 10 pages, 2015.

[30] B. Schilling, M. M. S. Bharath, R. H. Row et al., "Rapid purification and mass spectrometric characterization of mitochondrial NADH dehydrogenase (Complex I) from rodent brain and a dopaminergic neuronal cell line," Molecular and Cellular Proteomics, vol. 4, no. 1, pp. 84-96, 2005.

[31] H. Ding, C.-Z. Yan, H. Shi et al., "Hepcidin is involved in iron regulation in the ischemic brain," PLoS ONE, vol. 6, no. 9, Article ID e25324, 2011.
[32] F. Du, C. Qian, Z. M. Qian et al., "Hepcidin directly inhibits transferrin receptor 1 expression in astrocytes via a cyclic AMPprotein kinase a pathway," Glia, vol. 59, no. 6, pp. 936-945, 2011.

[33] A. Schober, "Classic toxin-induced animal models of Parkinson's disease: 6-OHDA and MPTP," Cell and Tissue Research, vol. 318, no. 1, pp. 215-224, 2004.

[34] K. L. Double, M. Maywald, M. Schmittel, P. Riederer, and M. Gerlach, "In vitro studies of ferritin iron release and neurotoxicity," Journal of Neurochemistry, vol. 70, no. 6, pp. 2492-2499, 1998.

[35] N. Song, J. Wang, H. Jiang, and J. Xie, "Ferroportin 1 but not hephaestin contributes to iron accumulation in a cell model of Parkinson's disease," Free Radical Biology and Medicine, vol. 48, no. 2, pp. 332-341, 2010.

[36] N. Song, H. Jiang, J. Wang, and J.-X. Xie, "Divalent metal transporter 1 up-regulation is involved in the 6-hydroxydopamineinduced ferrous iron influx," Journal of Neuroscience Research, vol. 85, no. 14, pp. 3118-3126, 2007.

[37] H. McGrath Jr. and P. G. Rigby, "Hepcidin: inflammation's iron curtain,” Rheumatology, vol. 43, no. 11, pp. 1323-1325, 2004.

[38] R. J. Basseri, E. Nemeth, M. E. Vassilaki et al., "Hepcidin is a key mediator of anemia of inflammation in Crohn's disease," Journal of Crohn's and Colitis, vol. 7, no. 8, pp. e286-e291, 2013.

[39] E. C. Hirsch, S. Vyas, and S. Hunot, "Neuroinflammation in Parkinson's disease," Parkinsonism and Related Disorders, vol. 18, supplement 1, pp. S210-S212, 2012.

[40] K. U. Tufekci, R. Meuwissen, S. Genc, and K. Genc, "Inflammation in Parkinson's disease," Advances in Protein Chemistry and Structural Biology, vol. 88, pp. 69-132, 2012.

[41] J. Bove, D. Prou, C. Perier, and S. Przedborski, “Toxin-induced models of Parkinson's disease," NeuroRx, vol. 2, no. 3, pp. 484494, 2005.

[42] R. Raha-Chowdhury, A. A. Raha, S. Forostyak, J.-W. Zhao, S. R. W. Stott, and A. Bomford, "Expression and cellular localization of hepcidin mRNA and protein in normal rat brain," $B M C$ Neuroscience, vol. 16, article 24, 2015. 


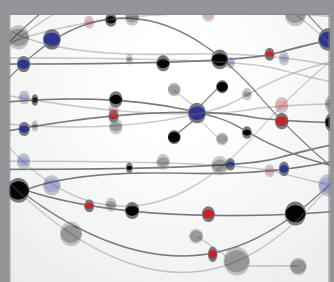

The Scientific World Journal
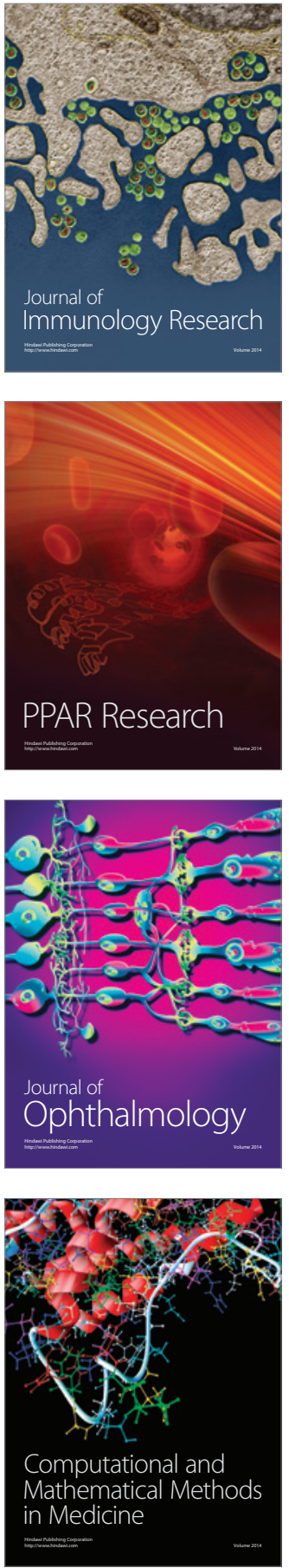

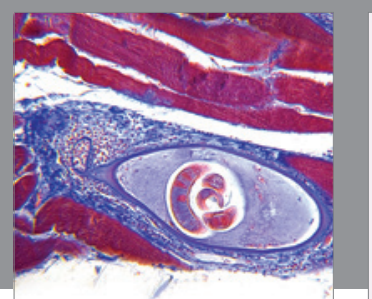

Gastroenterology Research and Practice

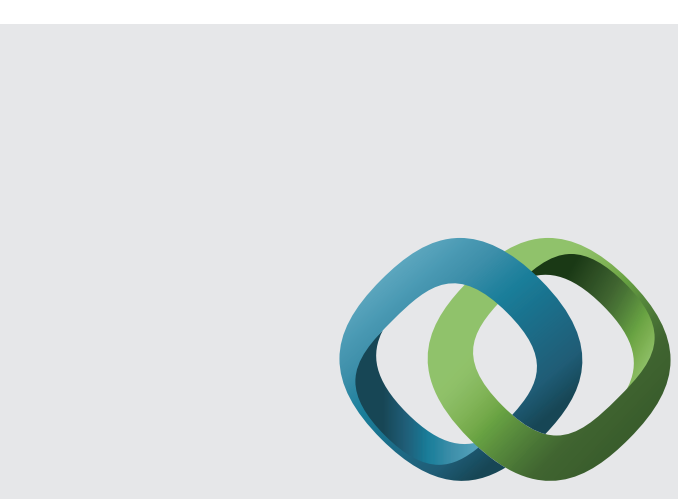

\section{Hindawi}

Submit your manuscripts at

http://www.hindawi.com
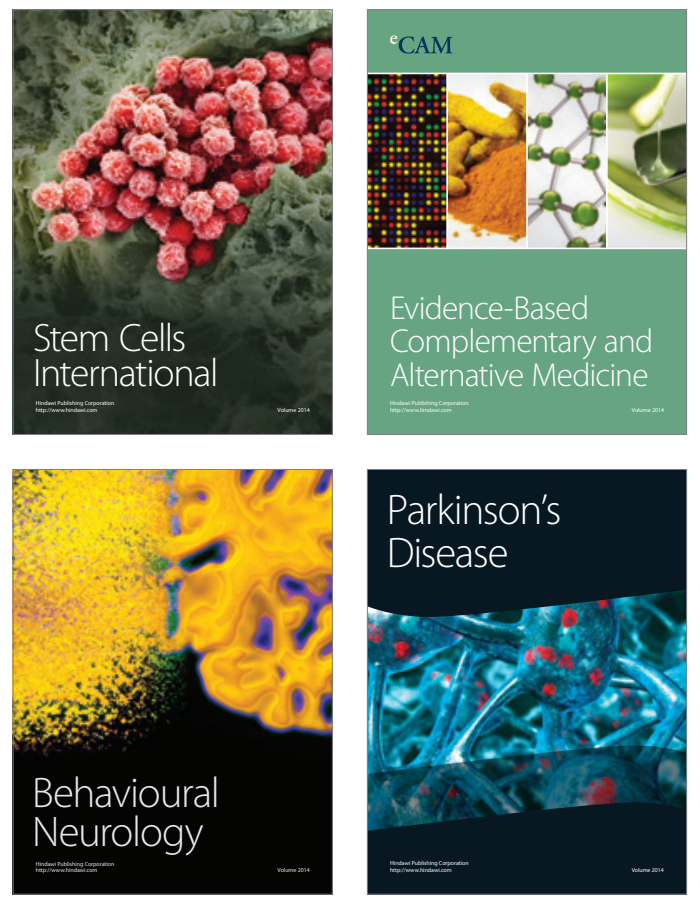
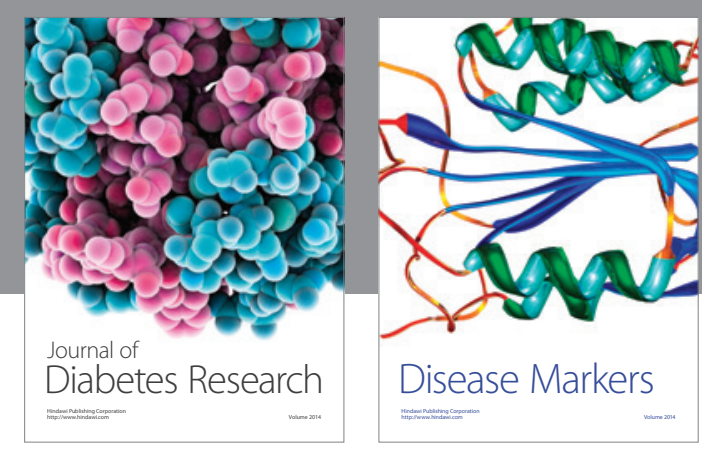

Disease Markers
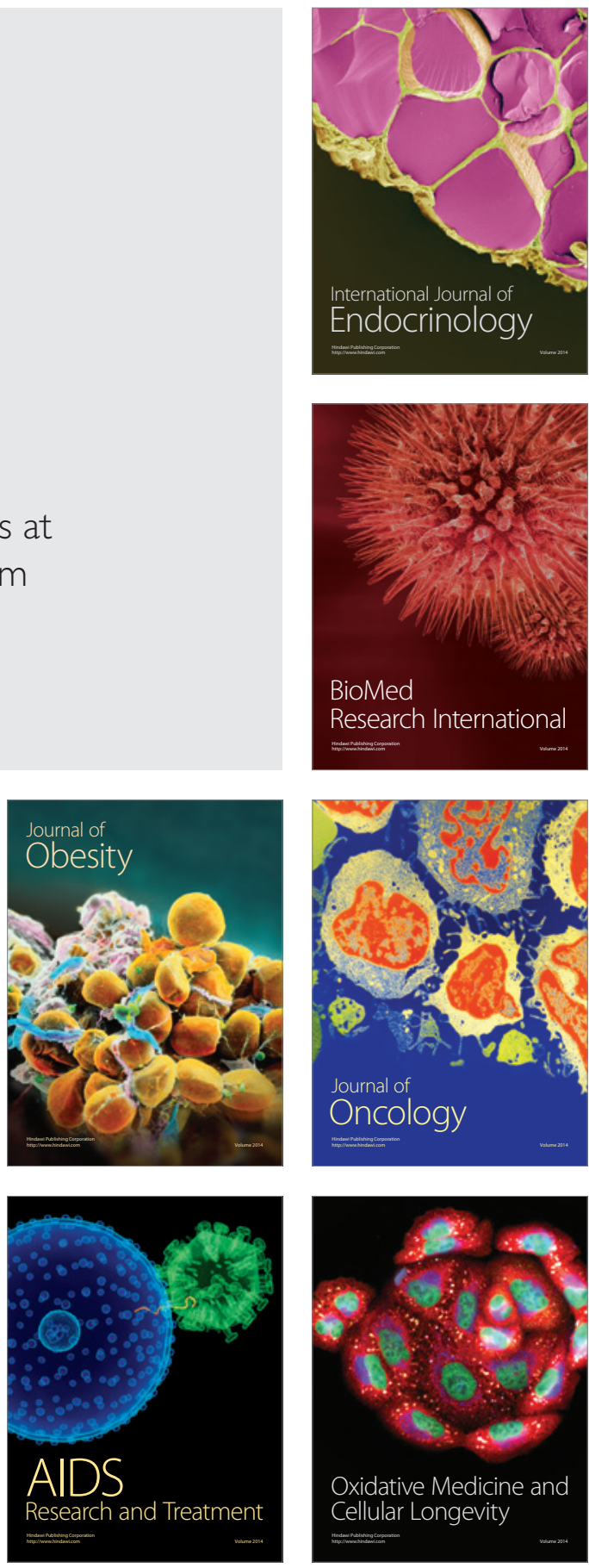